\title{
Catching Gall Bladder Cancer Early- Importance of Cholelithiasis and Radiologist
}

\section{ABSTRACT}

Introduction: Increased relative risk of Gall Bladder (GB) cancer in patients with gall stones has been reported in literature but there are few reports on nature of stones. However, it is difficult to predict which patient suffering from cholelithiasis and cholecystitis may develop or already has early stages of carcinoma

Aim: The present study was undertaken to assess the malignant potential and relevance of gall stones, age and gender in GB carcinoma to propose a screening protocol and early intervention for high risk patients.

Materials and Methods: This was a retrospective study done at Moti Lal Nehru Medical College, Prayagraj, Uttar Pradesh, India from May 2012 to May 2019. This study included 200 histopathologically- Fine Needle Aspiration Cytology (FNAC) or postoperative specimen biopsy proven GB carcinoma and 200 control patients with upper abdominal pain but without any evidence of neoplastic changes on USG. All cases and controls were subjected to USG evaluation first followed by plain CT scan with oral contrast only and Contrast Enhanced CT (CECT) scan of whole abdomen as per protocol. Scans were evaluated for detection of gall stone profile in terms of number (solitary/multiple), size (small/large) and density (radio-opaque/ radiolucent). The statistical analysis was done by using IBM Statistical Package for the Social Sciences (SPSS) version 18.0.
Results: Male to female ratio in this study was 1:2. GB calculi cannot be considered as bystander as overall $25 \%$ patients of both sexes had cholelithiasis. In the present study, 25\% who were having carcinoma GB have been detected to have gall stones and out of which $74 \%$ were having small calculi. The study showed the association between GB cancer and solitary and multiple large calculi over different age groups to be not statistically significant ( $p$-value $=0.3976 ; \chi^{2}=0.716$ ). Association of small and multiple calculi came out to be highly significantly associated with carcinoma in $67 \%$ of patients. Furthermore, it was observed that the association between multiple small calculi and GB cancer over the different age groups came out to be extremely statistically significant ( $p$-value $=0.0001 ; \chi^{2}=28.62$ ). The association between gall stones in GB cancer patients differ statistically significant over the age groups from cases with gall stones in control group ( $p$-value $=0.005497 ; \chi^{2}=7.7082$ ).

Conclusion: From the findings of the study author proposed screening via ultrasound and guided FNAC for females over 40 years of age with multiple small calculi not undergoing immediate cholecystectomy to detect early emergence of carcinoma. It is also suggested that clinicians should post patients with multiple, small GB calculi for cholecystectomy on a priority basis and histopathology should be done in each case of $\mathrm{GB}$ wall thickening.

\section{INTRODUCTION}

Gall bllader carcinoma being the most common malignancy of the biliary tract, ranks $6^{\text {th }}$ among gastrointestinal cancers worldwide $[1,2]$. Epidemiological studies in India report its prevalence to range from 0.1-3.7 per 100,000 population for males to 0.3-8.9 per 100,000 population for females [3]. However, the magnitude of the problem could be huge especially in the endemic zones of North-east India (Western Bihar and Eastern Uttar Pradesh) where it is the third commonest malignancy of the alimentary tract $[4,5]$.

The median age at presentation is considered to be 67 years [2]. Menopausal females and smokers are considered at higher risk of GB cancer as per various epidemiological studies $[6,7]$. Cholelithiasis is a well-established risk factor for the development of GB carcinoma [8], and gallstones are present in 74\%-92\% of affected patients [9].

The GB carcinoma is generally diagnosed incidentally when patients present symptoms of abdominal pain, nausea, vomiting and/ or fever related to co-existent cholelithiasis or cholecystitis. One percent of patients undergoing cholecystectomy for cholelithiasis have an incidental GB carcinoma $[10,11]$. The majority of patients with GB carcinoma present with advanced disease. Symptoms are typically indolent. Chronic abdominal pain, anorexia, or weight loss is common initial complaints [2]. Physical examination may demonstrate a lump, hepatomegaly and jaundice [12]
Increased relative risk of GB cancer in patients with gall stones has been reported but there are few reports on nature, density and number of stones affecting prognosis $[7,13]$. Keeping this in mind, there is a need to further study the risk of GB cancer with respect to number, size and type of gall stones. It is difficult to predict which pateint is suffering from cholelithiasis and cholecystitis may develop or already has early stages of carcinoma. A wide array of medical, conservative and surgical approaches is present regarding management of cholelithiasis and cholecystitis. But in absence of a uniform fixed protocol regarding early prediction and preventive surgical management of such patients more prone to develop carcinoma, majority of GB carcinomas are detected late. Hence, this study was conducted to assess the malignant potential of different types and numbers of gall stones via ultrasonography, plain CT with oral contrast and CECT. The present study was undertaken to know the relevance of calculus, age and gender in prediction of GB carcinoma to propose a screening protocol and early intervention for high risk patients.

\section{MATERIALS AND METHODS}

This was a retrospective study done at Moti Lal Nehru Medical College, Prayagraj, Uttar Pradesh India from May 2012 to May 2019. Approval for this study was obtained by Ethical Committee of MLN Medical College, Prayagraj, Uttar Pradesh, India. 
Inclusion and Exclusion criteria: 200 patients with histopathologically (FNAC or postoperative specimen biopsy) proven GB carcinoma and 200 control patients with upper abdominal pain but without any evidence of neoplastic changes on USG were included. Subjects below 18 years of age, cases with previous known malignancy or metastasis of other origin apart from GB cancer were excluded.

\section{Study Procedure}

All cases and controls were subjected to USG evaluation first followed by plain CT scan (with oral contrast only) and CECT scan of whole abdomen as per protocol. USG examination was performed using LOGIQ- P9 USG machine (GE Healthcare). CECT Scan was done on Revolution ACTs (GE Healthcare). Initially plain axial scans were taken from the domes of diaphragm till the level of iliac crest, after administering $1000 \mathrm{~mL}$ of oral iodinated water soluble contrast $(20 \mathrm{~mL}$ of $350 \mathrm{mgl} / \mathrm{mL}$ non ionic water soluble contrast mixed with water to make $1000 \mathrm{~mL}$ ) with $800 \mathrm{~mL}$ half an hour before examination and $200 \mathrm{~mL}$ at the time of examination for bowel opacification. Scanning was performed using pitch of 1.0:1, a scanning time of $1.0 \mathrm{sec} /$ rotation, table speed of $10 \mathrm{~mm} /$ rotation, 160-180 mAs, and $120 \mathrm{kVp}$. Later on contrast enhanced scan was done after administering $80-100 \mathrm{~mL}$ of non ionic water soluble contrast $(350 \mathrm{mgl} / \mathrm{mL})$ depending upon weight of the patient with pressure injector at a rate of $2.0 \mathrm{~mL} / \mathrm{sec}$ and scans were taken in portal venous phase (50-55 sec after contrast initiation of contrast administration). Images were acquired with slice thickness of $5 \mathrm{~mm}$ and reconstruction interval of $0.7 \mathrm{~mm}$ followed by Multiplanar Reconstruction (MPR) algorithm both in coronal and sagittal plane. Scans were evaluated for detection of gall stone profile in terms of number (solitary/multiple), size (small/large) and density (radioopaque/radiolucent). Size of $10 \mathrm{~mm}$ was the cutoff for small and large calculi. Criteria for radio-opaque stones were stones which were hyperdense to bile on CT scan and for radiolucent stones were hyperechoic on USG but isodense to bile on CT scan and hence imperceptible on CT Scan.

\section{STATISTICAL ANALYSIS}

The statistical analysis was done using IBM SPSS version 18.0. The statistical test applied here was Chi-square two-tailed test for association with and without Yates correction as required at 5\% level of significance or 95\% confidence interval.

\section{RESULTS}

Female to male ratio in the present study was 2:1. GB calculi cannot be considered as bystander as overall $25 \%$ patients of both sexes had cholelithiasis [Table/Fig-1]. However, few patients had carcinoma GB without any evidence of calculus [Table/Fig-2]. Total of 50 patients (25\%) who were having carcinoma GB have been detected to have gall stones and out of which 37 patients (74\%) were having small calculi [Table/Fig-3,4]. Out of 200 patients, 6.5\% had large calculi [Table/Fig-5]. At 5\% level of significance, Chi-square without Yates correction two-tailed test showed the association between solitary and multiple large calculi over different age groups to be not statistically significant ( $p$-value=0.3976; $\chi^{2}=0.716$ ) [Table/Fig-6].

\begin{tabular}{|l|c|c|c|c|}
\hline \multirow{2}{*}{ Age interval } & \multicolumn{4}{|c|}{ Gall stone } \\
\cline { 2 - 5 } & Present & $\%$ & Absent & $\%$ \\
\hline 30 and below & 2 & 1 & 5 & 2.50 \\
\hline $31-40$ & 7 & 3.50 & 27 & 13.50 \\
\hline $41-50$ & 12 & 6.00 & 34 & 17 \\
\hline $51-60$ & 11 & 5.50 & 35 & 17.50 \\
\hline $61-70$ & 13 & 6.50 & 31 & 15.50 \\
\hline 71 and above & 5 & 2.50 & 18 & 9 \\
\hline Total & 50 & 25 & 150 & 75 \\
\hline
\end{tabular}

[Table/Fig-1]: Distribution of gall stones according to age in 200 cases of Gall Bladder (GB) cancer patients.
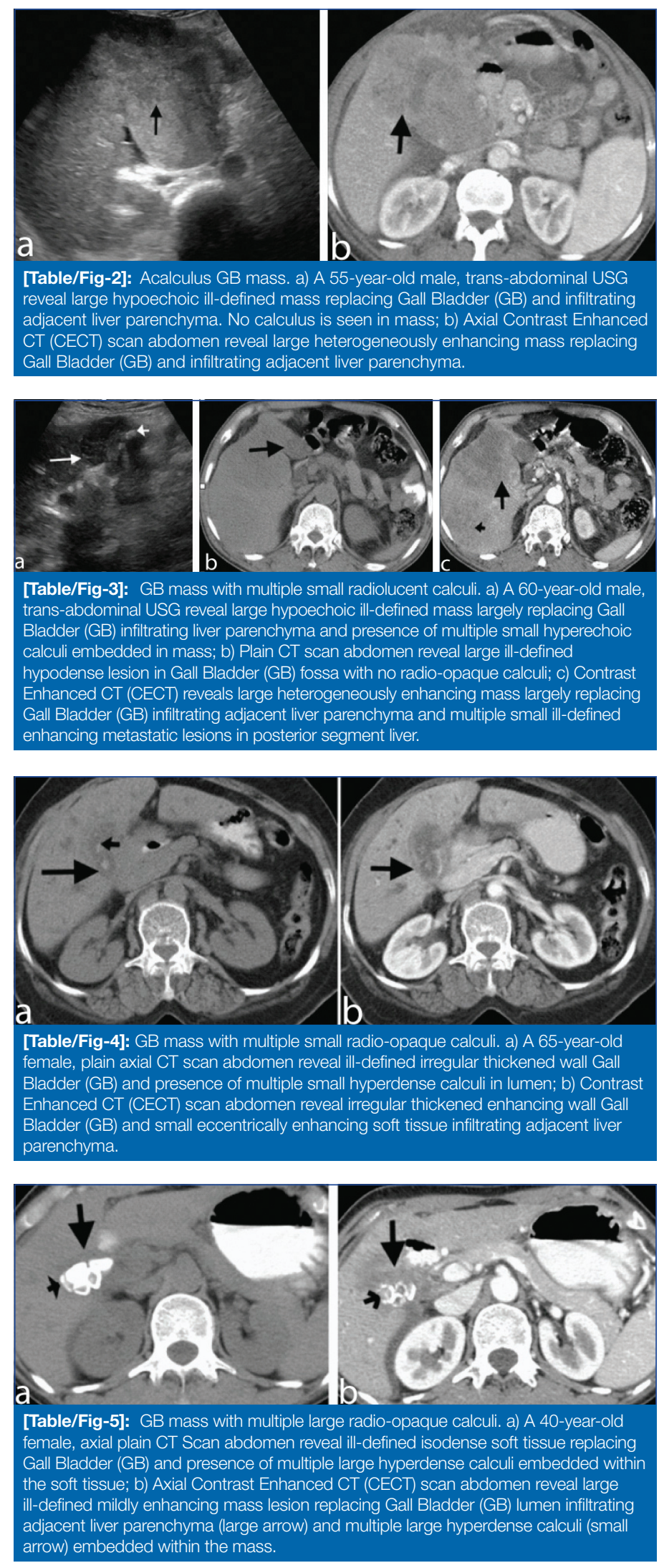

In [Table/Fig-7], Chi-square without Yates correction two-tailed test showed the association between multiple small calculi and GB cancer over the different age groups to be extremely statistically significant ( $p$-value $=0.0001 ; \chi^{2}=28.62$ ). None of patients had radiolucent solitary small calculus and only $2 \%$ of total of 200 patients had radiopaque solitary calculus [Table/Fig-6-8].

In control group of 200 patients with upper abdominal pain or jaundice without GB cancer, 19 had cholelithiasis. [Table/Fig-9] showed that the percent of cases with gall stones in GB cancer patients differ from percent of cases with gall stones in control group and this difference was found to be statistically significant $\left(p=0.005497 ; \chi^{2}=7.7082\right)$. 


\begin{tabular}{|c|c|c|c|c|c|c|c|c|c|}
\hline \multirow{3}{*}{$\begin{array}{l}\text { Age } \\
\text { interval }\end{array}$} & \multicolumn{4}{|c|}{ Solitary calculus } & \multicolumn{4}{|c|}{ Multiple calculi } & \multirow{3}{*}{$\begin{array}{l}\mathrm{p} \text {-value } \\
\text { and Chi- } \\
\text { square } \\
\text { value }\end{array}$} \\
\hline & \multicolumn{2}{|c|}{ Radiolucent } & \multicolumn{2}{|c|}{ Radio-opaque } & \multicolumn{2}{|c|}{ Radiolucent } & \multicolumn{2}{|c|}{ Radio-opaque } & \\
\hline & No. & $\%$ & No. & $\%$ & No. & $\%$ & No. & $\%$ & \\
\hline $\begin{array}{l}30 \text { and } \\
\text { below }\end{array}$ & 0 & 0 & 0 & 0 & 1 & 2 & 0 & 0 & \multirow{7}{*}{$\begin{array}{c}0.3976 \\
0.716\end{array}$} \\
\hline $31-40$ & 0 & 0 & 0 & 0 & 0 & 0 & 0 & 0 & \\
\hline $41-50$ & 0 & 0 & 0 & 0 & 2 & 4 & 2 & 4 & \\
\hline $51-60$ & 1 & 2 & 1 & 2 & 1 & 2 & 1 & 2 & \\
\hline $61-70$ & 1 & 2 & 0 & 0 & 1 & 2 & 0 & 0 & \\
\hline $\begin{array}{l}71 \text { and } \\
\text { above }\end{array}$ & 2 & 4 & 0 & 0 & 0 & 0 & 0 & 0 & \\
\hline Total & 4 & 8 & 1 & 2 & 5 & 10 & 3 & 6 & \\
\hline \multicolumn{10}{|c|}{$\begin{array}{l}\text { [Table/Fig-6]: Relationship of age to large gall stone }(\geq 10 \mathrm{~mm}) \text { characteristic in } \\
200 \text { Gall Bladder (GB) cancer patients. } \\
\text { Chi-square without Yates correction two-tailed test used at } 5 \% \text { level of significance }\end{array}$} \\
\hline
\end{tabular}

\begin{tabular}{|c|c|c|c|c|c|c|c|c|c|}
\hline \multirow[b]{3}{*}{ Age interval } & \multicolumn{4}{|c|}{ Solitary calculus } & \multicolumn{4}{|c|}{ Multiple calculi } & \multirow{3}{*}{$\begin{array}{l}p \text {-value } \\
\text { and Chi } \\
\text { square } \\
\text { value }\end{array}$} \\
\hline & \multicolumn{2}{|c|}{ Radiolucent } & \multicolumn{2}{|c|}{ Radio-opaque } & \multicolumn{2}{|c|}{ Radiolucent } & \multicolumn{2}{|c|}{ Radio-opaque } & \\
\hline & No. & $\%$ & No. & $\%$ & No. & $\%$ & No. & $\%$ & \\
\hline 30 and below & 0 & 0 & 0 & 0 & 1 & 2 & 0 & 0 & \multirow{7}{*}{$\begin{array}{l}0.0001 \\
28.62\end{array}$} \\
\hline $31-40$ & 0 & 0 & 1 & 2 & 0 & 0 & 6 & 12 & \\
\hline $41-50$ & 0 & 0 & 0 & 0 & 2 & 4 & 6 & 12 & \\
\hline $51-60$ & 0 & 0 & 2 & 4 & 1 & 2 & 4 & 8 & \\
\hline $61-70$ & 0 & 0 & 0 & 0 & 1 & 2 & 10 & 20 & \\
\hline 71 and above & 0 & 0 & 0 & 0 & 0 & 0 & 3 & 6 & \\
\hline Total & 0 & 0 & 3 & 6 & 5 & 10 & 29 & 58 & \\
\hline \multicolumn{10}{|c|}{$\begin{array}{l}\text { [Table/Fig-7]: Relationship of age to small gall stone }(<10 \mathrm{~mm}) \text { characteristic in } \\
200 \text { Gall Bladder (GB) cancer patients. } \\
\text { Chi-square without Yates correction two-talled test used at } 5 \% \text { level of significance }\end{array}$} \\
\hline
\end{tabular}

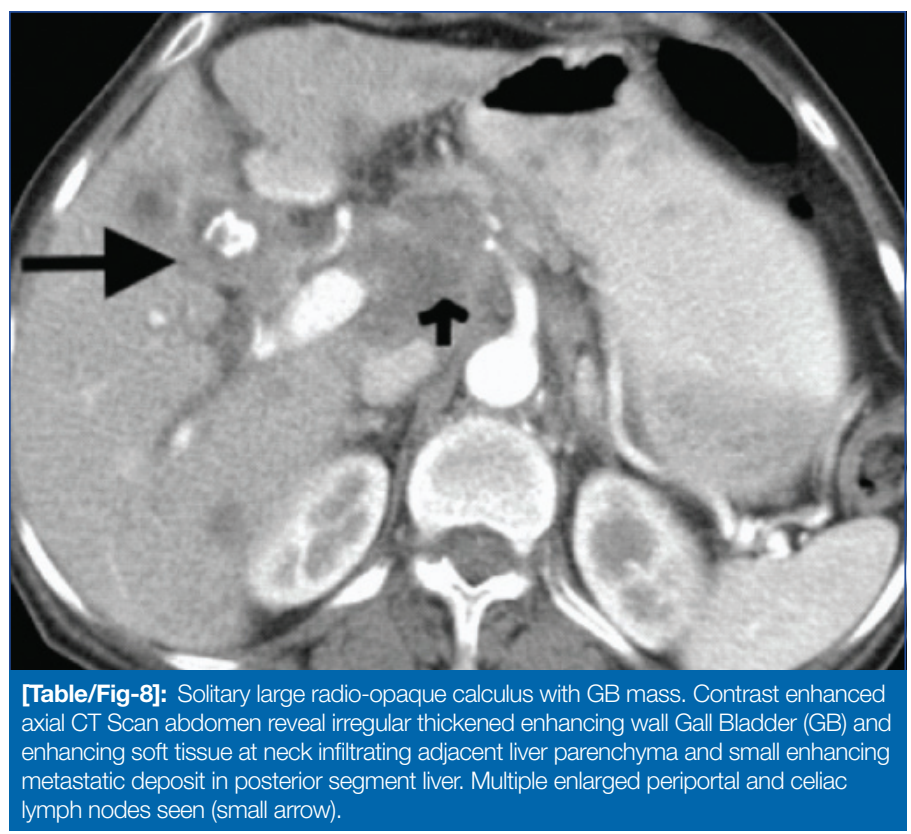

\begin{tabular}{|c|c|c|c|c|}
\hline Cholelithiasis & $\begin{array}{l}\text { GB cancer } \\
\text { (cases) }\end{array}$ & $\begin{array}{l}\text { GB cancer } \\
\text { (controls) }\end{array}$ & Total & $\begin{array}{c}p \text {-value and } \\
\text { Chi-square value }\end{array}$ \\
\hline Present & 50 & 19 & 78 & \multirow{3}{*}{$\begin{array}{c}0.005497 \\
7.7082\end{array}$} \\
\hline Absent & 150 & 181 & 322 & \\
\hline Total & 200 & 200 & 400 & \\
\hline
\end{tabular}

\section{DISCUSSION}

It has been postulated that chronic irritation and inflammation of GB wall with gall stone leads to mucosal dysplasia and is subsequently associated with increased risk of GB carcinoma [14]. Gallstones can be classified as cholesterol, mixed, or pigment stones. In western countries, approximately $75 \%$ of GB stones are of cholesterol stones [15]. Detectability of gallstones on CT is affected by their chemical composition. The amount of calcium phosphate or calcium carbonate within gallstones correlates with their visibility on CT. Pigment stones have a higher affinity for calcium carbonate and calcium bilirubinate and generally have higher CT attenuation values. Pure cholesterol stones on the other hand, are lower in attenuation and not as readily detected by CT [15-18].

Regarding ultrasonographic characteristics of gallstones, sonographic criteria for cholesterol stones are stones that float in the GB or stones that produce acoustic shadows without internal echoes from the stones [9]. In a study conducted by Good LI et al., no correlation was found between gallstone type or calcium content and acoustic shadowing. Acoustic shadowing was found to be related to the size of the gallstone. Stones $4 \mathrm{~mm}$ or greater in diameter are much more likely to produce distinct sonic shadows compared to smaller stones regardless of composition [19].

In the present study, gall stones were present in $25 \%$ of GB carcinoma patients as compared to $74 \%-92 \%$ in earlier studies $[2,20]$. This statistic in earlier studies may be inflated, as those undergoing cholecystectomy for cholelithiasis are more likely to be diagnosed incidentally or postoperatively with GB cancer. Also, obesity, being a major factor in formation of cholesterol gall stones is still at a lower prevalence in developing countries. Thus, ethnical and geographical based variations must be kept in mind while assessing incidence of GB cancer in patients of cholelithiasis. This may be also be due to other predisposing factors such as chronic Salmonella type infection, exposure to chemicals used in the rubber, automobile, wood finishing, and metal fabricating industries which lead to GB carcinoma apart from gall stones in our studied population [21-23].

Shrikhande SV et al., in their study concluded that in view of lack of absolute proof, cholelithiasis is a co-factor the causation of GB carcinoma [20]. They recommended tailoring of prophylactic cholecystectomy. In the present study, it was found that patients with solitary large calculus were not significantly associated with GB cancer. Hence, not all patients need to undergo prophylactic cholecystectomy.

Most of gall stones in carcinoma patients were found to be in $5^{\text {th }}-7^{\text {th }}$ decades of age group with a preponderance of female gender. Female to male ratio in the present study was $2: 1$ while it was found to be three times more common in females in earlier studies [2,24,25]. Prevalence of fasting on religious grounds in in Northern India might result in increase in presence of small calculi in concentrated bile in both genders which might have led to lesser variation in gender ratio. Presence of small (less than $10 \mathrm{~mm}$ ) and multiple calculi came out to be highly significantly associated with GB carcinoma. Hence, screening via ultrasound and guided FNAC is proposed for females over 40 years of age with multiple small calculi not undergoing immediate cholecystectomy to detect early emergence of carcinoma. We also suggest to urge the clinicians to post patients with multiple, small GB calculi for cholecystectomy on a priority basis and histopathology should be done in each case of GB wall thickening (or cholecystectomy).

\section{Limitation(s)}

The main limitation of the study was that the predisposing factors for GB cancer other than gall stones were not ruled out from study population. Also, the present study was a single centre study. Further studies with a larger sample size should be conducted.

\section{CONCLUSION(S)}

The GB carcinoma along with cholelithiasis is present mostly in $5^{\text {th }}$ to $7^{\text {th }}$ decades of age groups with a preponderance of female gender. Presence of small, multiple GB calculi are highly significantly associated with GB carcinoma. The authors propose ultrasonography screening for females over 40 years of age with multiple small calculi to detect early carcinoma. They also suggest to urge the clinicians to consider patients with multiple, small GB calculi for cholecystectomy and histopathology. 


\section{Acknowledgement}

The authors would like to acknowledge contribution of residents, staff and consultants of Department of Surgery, MLNMC, Prayagraj, UP, for their support in patient management. Our heartfelt acknowledgement to Department of Pathology, MLNMC, Prayagraj, UP, for their insights in histopathological review.

\section{REFERENCES}

[1] Lazcano-Ponce EC, Miquel JF, Muñoz N, Herrero R, Ferric C, Wistuba II, et al. Epidemiology and molecular pathology of gallbladder cancer. CA Cancer $\mathrm{J}$ Clin. 2001;51:349-64.

[2] Hundal R, Shaffer EA. Gallbladder cancer: Epidemiology and outcome. Clin Epidemiol. 2014:6:99-109.

[3] Indian Council of Medical Research. Consolidated Report of the Population Based Cancer Registries of the National Cancer Registry Programme (1990-1996). New Delhi: ICMR Publication; 2001. Pp. 52.

[4] Shukla VK, Khandelwal C, Roy SK, Vaidya MP. Primary carcinoma of the gall bladder: A review of a 16-year period at the university hospital. J Surg Oncol. 1985;28:32-35.

[5] Pandey M, Pathak AK, Gautam A, Aryya NC, Shukla VK. Carcinoma of the gallbladder: A retrospective review of 99 cases. Dig Dis Sci. 2001;46:1145-51.

[6] Singh BP, Khan WF, Rathore YS, Pol MM. Incidental carcinoma gallbladder: Incidence, risk factors, and factors affecting survival-5-year experience from a tertiary care institute. Journal of Gastrointestinal Cancer. 2020;51(3):980-87.

[7] Khan ZR, Neugut Al, Ahsan H, Chabot JA. Risk factors for biliary tract cancers. Am J Gastroenterol. 1999;94:149-52.

[8] Kim YS. Epidemiology and risk factors of gallbladder cancer. The Korean Journal of Pancreas and Biliary Tract. 2018;23(1):07-14

[9] Andriulli A, Gremo L, Limerutti G, Bianchi A, Berti E, Arrigoni A, et al. Prediction of gallstone compostion by ultrasound: Implications for non-surgical therapy. J. Stone Dis. 1993;5(2):96-104.

[10] Wanebo HJ, Vezeridis MP. Carcinoma of the gallbladder. J Surg Oncol Suppl. 1993;3:134-39

[11] Panebiano A, Laforgia R, Volpi A. Predictive factors for incidental gallbladder cancer (IGBC) in patients undergoing cholecystectomy for presumed benign disease. A single-center experience. Ann Ital Chir. 2018;89(2):118-27.
[12] Kai K, Aishima S, Miyazaki K. Gallbladder cancer: Clinical and pathological approach. World Journal of Clinical Cases. 2014;2(10):515-21.

[13] Roa I, Ibacache G, Roa J, Araya J, De Aretxabala X, Muñoz S. Gallstones and gallbladder cancer-volume and weight of gallstones are associated with gallbladder cancer: A case-control study. Journal of Surgical Oncology. 2006;93(8):624-28.

[14] Solaini L, Sharma A, Watt J, losifidou S, Chin Aleong JA, Kocher HM. Predictive factors for incidental gallbladder dysplasia and carcinoma. J Surg Res. 2014;189(1):17-21.

[15] Bortoff GA, Chen MY, Ott DJ, Wolfman NT, Routh WD. Gallbladder stones: Imaging and intervention. Radiographics. 2000;20(3):751-66.

[16] Lee JK, Kim TK, Byun JH, Kim AY, Ha HK, Kim PN, et al. Diagnosis of intrahepatic and common duct stones: Combined unenhanced and contrast-enhanced helical CT in 1090 patients. Abdom Imaging. 2006;31:425-32.

[17] Kalra N, Gupta P, Singhal M, Gupta R, Gupta V, Srinivasan R, et al. Crosssectional imaging of gallbladder carcinoma: An update. Journal of Clinical and Experimental Hepatology. 2019;9(3):334-44.

[18] John S, Moyana T, Shabana W, Walsh C, Mclnnes MD. Gallbladder cancer: Imaging appearance and pitfalls in diagnosis. Canadian Association of Radiologists Journal. 2020;71(4):448-58.

[19] Good LI, Edell SL, Soloway RD, Trotman BW, Mulhern C, Arger PA. Ultrasonic properties of gallstones: Effect of stone size and composition. Gastroenterology. 1979;77(2):258-63.

[20] Shrikhande SV, Barreto SG, Singh S, Udwadia TE, Agarwal AK. Cholelithiasis in gallbladder cancer: Coincidence, cofactor, or cause! Eur J Surg Oncol. 2010;36(6):514-19.

[21] Tyagi BB, Manoharan N, Raina V. Risk factors for gallbladder cancer: A population based case-control study in Delhi. Indian Journal of Medical and Paediatric Oncology. 2008;29(1):16.

[22] Bond GG, McLaren EA, Sabel FL, Bodner KM, Lipps TE, Cook RR. Liver and biliary tract cancer among chemical workers. Am J Ind Med. 1990;18:19-24.

[23] Dutta U, Bush N, Kalsi D, Popli P, Kapoor VK. Epidemiology of gallbladder cancer in India. Chin Clin Oncol. 2019;8(4):33.

[24] Rawla P, Sunkara T, Thandra KC, Barsouk A. Epidemiology of gallbladder cancer. Clinical and Experimental Hepatology. 2019:5(2):93.

[25] Kanthan R, Senger JL, Ahmed S, Kanthan SC. Gallbladder cancer in the $21^{\text {st }}$ century. Journal of Oncology. 2015;2015:967472.

\section{PARTICULARS OF CONTRIBUTORS:}

1. Associate Professor, Department of Radiodiagnosis, Moti Lal Nehru Medical College, Prayagraj, Uttar Pradesh, India.

2. Senior Resident, Department of Radiodiagnosis, Moti Lal Nehru Medical College, Prayagraj, Uttar Pradesh, India.

3. Senior Professor and Head, Department of Radiotherapy and Oncolgy, Moti Lal Nehru Medical College, Prayagraj, Uttar Pradesh, India.

4. Assistant Professor, Department of Community Medicine, Moti Lal Nehru Medical College, Prayagraj, Uttar Pradesh, India.

NAME, ADDRESS, E-MAIL ID OF THE CORRESPONDING AUTHOR:

Mrityunjay Nursing Home, Teliarganj, Prayagraj, Uttar Pradesh, India.

E-mail: drasthasingh@outlook.com
Dr. Astha Singh,
PLAGIARISM CHECKING METHODS: [Jain Het al.]

- Plagiarism X-checker: Dec 12, 2020

- Manual Googling: Mar 03, 2021

- iThenticate Software: Mar 05, 2021 (5\%)
ETYMOLOGY: Author Origin

\section{AUTHOR DECLARATION:}

- Financial or Other Competing Interests: None

- Was Ethics Committee Approval obtained for this study? Yes

- Was informed consent obtained from the subjects involved in the study? Yes

- For any images presented appropriate consent has been obtained from the subjects. Yes
Date of Submission: Oct 09, 2020 Date of Peer Review: Feb 05, 2021 Date of Acceptance: Mar 05, 2021 Date of Publishing: Oct 01, 2021 